Serge Kakou, Découverte photographique de la Nouvelle-Calédonie 1848-1900

1998, Actes Sud, Arles

\title{
Emmanuelle Crane
}

\section{(2) OpenEdition}

\section{Journals}

Édition électronique

URL : http://journals.openedition.org/jso/1747

DOI : $10.4000 /$ jso. 1747

ISSN : $1760-7256$

Éditeur

Société des océanistes

Édition imprimée

Date de publication : 1 juin 2001

Pagination : 96-97

ISSN : 0300-953x

Référence électronique

Emmanuelle Crane, «Serge Kakou, Découverte photographique de la Nouvelle-Calédonie 1848-1900», Journal de la Société des Océanistes [En ligne], 112 | Année 2001-1, mis en ligne le 28 mai 2008,

consulté le 24 septembre 2020. URL : http://journals.openedition.org/jso/1747 ; DOI : https://doi.org/ $10.4000 / j s 0.1747$ 
cette mise à l'écart — fut-elle choisie — qui explique les attaques et critiques très sévères portées à l'encontre de nombreux responsables politiques kanak, pour certains aujourd'hui disparus... Quelles que soient les divergences politiques et les rancœurs de Gabriel Païta envers ses anciens compagnons de route, on ne peut que regretter ses mises en cause extrêmement graves et le dénigrement de ceux qui ne sont plus là pour répondre. Le témoignage de Gabriel Païta sur cette période dramatique de l'histoire calédonienne n'est pas crédible, tant la partialité y est de mise. Exclu du mouvement par lequel il était entré en politique, Gabriel Païta créera le Parti fédéral kanak puis, en 1985, l'OPAO.

L'aspect « règlement de comptes » que le récit prend au fil des pages aurait dû alerter ces jeunes auteurs historiens, qui auraient pu éviter les pièges de l'amalgame s'ils avaient pris soin de confronter le plus objectivement possible les faits historiques aux interprétations subjectives de leur interviewé.

Aussi est-il nécessaire de lire ce récit avec un œil très critique!

\section{Isabelle LEBLIC, LACITO-CNRS.}

Kakou Serge, 1998. Découverte photographique de la Nouvelle-Calédonie 1848-1900, Actes Sud, Arles.

Avec plus de 170 illustrations, Serge Kakou nous fait découvrir la Nouvelle-Calédonie du $\mathrm{XIX}^{\mathrm{e}}$ siècle sous tous ses angles photographiques. Cet ouvrage, d'une richesse de clichés extraordinaire, regroupe les photographes les plus connus et anciens de Hughan à Robin. Les thèmes exploités à cette époque par la photographie sont, sans aucun doute, représentés ici. Ils nous sont rapportés accompagnés d'un texte clair et précis relatant les épisodes historiques qui s'y attachent.

Kakou aborde ces thèmes d'une manière chronologique, les divisant en sections qui n'ont apparemment pas de raison d'être si ce n'est que celle de sélectionner les dates de l'activité photographique de chaque artiste (mais il n'y a pas de suite logique entre leurs œuvres respectives). L'auteur essaie pourtant d'apporter un complément aux photographies choisies en précisant certains faits majeurs de l'histoire calédonienne, tout en décrivant le développement scientifique de la photographie ; il nous permet ainsi de mieux saisir l'évolution de la qualité photographique. Les clichés daguerréotypes se voient remplacés, après un succès long de douze ans, par la technique — plus rapide, moins coûteuse, aisément multipliable et pourtant tout aussi précise - du processus négatif-positif, ce dernier succédant à la plaque argentée au cours de la décennie $1850^{4}$.

Ce livre se veut avant tout un livre d'images, avec des reproductions allant d'un huitième à une double page pour une mise en valeur totale du sujet photographié. C'est donc le plaisir de l'image qui prime, le texte étant avant tout là pour approfondir l'illustration. Il est dommage que dans ce livre « esthétique » soient incorporées des lettres d'époque imprimées dans une typographie similaire au reste du texte, amoindrissant par là même leur mise en valeur. La «copie d'époque » doublée de ces photographies aurait laissé émaner un parfum d'ambiance $\mathrm{XIX}^{\mathrm{e}}$ siècle plus envoûtant que celui que l'on ressent en feuilletant le livre. Bien que le texte montre une longue recherche progressivement accumulée, Kakou ne va pas en profondeur dans son analyse photographique.

Il est évident que chaque cliché réalisé par le photographe ne représente pas forcément la réalité, sinon sa propre réalité, son interprétation personnelle. La photographie prise par Chapuy (p. 25) par exemple, qui représente le portrait du Père Goujon, pourrait être interprétée comme le missionnaire évoquant l'ange gardien et entouré de ses fidèles enfants indigènes et porteur de bonne nouvelle, le voilà comme « l'auréole du siècle des martyrs ${ }^{5}$. Une interprétation que l'on peut déduire en se référant à la composition de l'image (sujet, point de vue, cadrage, lumière, pose et profondeur de champ). Pourtant, Kakou ne discute pas le point de vue de l'artiste, ni d'ailleurs le sien, dans sa sélection d'images. En d'autres termes, l'auteur ne nous donne aucune référence sur ses croyances, mais seulement une perception globale et n'apporte que rarement sa propre interprétation. En voulant présenter une palette de thèmes dont les photos sélectionnées en font l'écho, Kakou nous communique une « description » de la Nouvelle-Calédonie plutôt que son « interprétation ».

Serge Kakou nous rapporte, par le biais du Père O'Reilly, que Néthing aurait dit que « le Kanak aime à se faire photographier ; ce que cherche le Kanak de 1890 c'est être exposé, encadré, sous la véranda de mon magasin, tout près de la place des cocotiers où le dimanche se rassemble la population » (p. 147). Cette opinion vaudrait un commentaire de la part de l'auteur mais il ne s'y risque pas. Une interprétation provenant de la communauté mélanésienne aurait pu éclaircir cette relation entre le photographe et son sujet ; entre le colonisateur et le colonisé ( $c f$. Portraits kanak, Paroles kanak).

Il aurait été intéressant que l'auteur inclut des images colorées ou imprimées avec d'autres tons pour couper un peu la répétition de l'image sépia, d'autant plus que ces photographies d'époque sont souvent prises à une certaine distance des sujets et donnent rapidement à l'ensemble une certaine monotonie. Il semble que la majorité des photographies (ou sinon toutes) n'ait point été retouchée pour l'impression et que Kakou ait voulu reproduire les clichés dans leur état actuel sans leur apporter de modifications permettant une meilleur image. À ce sujet, on note tout particulièrement le cliché panoramique de Nouméa, très abîmé, pris par Robin. Est-ce par souci de conserver ce sentiment de photos anciennes que l'auteur a décidé de le faire publier sans retouche? Si tel est le cas, on peut difficilement parler d'un livre d'art, car les 
photos auraient dû être imprimées et publiées après retouche afin d'obtenir une image plus proche de la perfection, afin d'accentuer le côté artistique de l'œuvre. Mais veut-il vraiment que son œuvre soit cataloguée comme livre d'Art ou comme livre «des photographies d'Art dans les années $1800 » ?$

Il est également intéressant de replacer le rôle du photographe dans le courant du XIX ${ }^{\mathrm{e}}$ siècle. L'époque voit en lui un héros moderne de la science et un héros de la magie dans le pays visité. Son rôle ne semble ni de dénoncer, ni de témoigner, mais plutôt d'informer et de répertorier un monde où tout change très vite. Il est évident que les « photos studio » représentent déjà un monde disparu et artificiel où l'on fait poser des Mélanésiens en tenue de « sauvage » pour satisfaire l'image que l'on s'en fait en Europe mais qui, bien entendu, ne représente plus le Kanak des environs de Nouméa. On peut d'ailleurs constater que les modèles des photographes gardèrent la même pose durant quatre générations, dans un décor préfabriqué et stéréotypé. Il est à noter, par ailleurs, que ces clichés servirent plus tard lorsque les colons donnèrent de leurs nouvelles en envoyant des cartes postales par la poste.

Serge Kakou fait une bonne analyse des motivations qui entraînent ces photographes d'époque à se lancer dans cette science, qui reste, à l'heure actuelle, toujours en évolution. L'auteur n'oublie pas de nous rappeler que cette science et technique coûtent cher et que l'enthousiasme ne suffit pas toujours. En effet, à une époque où peu de personnes ne pouvaient ni s'acheter le matériel adéquat ni avoir les connaissances pour le développement des clichés, la demande variait selon les années, mais le premier client restait essentiellement l'administration, ce qui provoqua, par exemple, la joie de Devambez lorsqu'il fut sollicité pour des prises de vue destinées à présenter «les multiples aspects de la colonie aux visiteurs de l'Exposition universelle de Paris en 1889 »(p. 116).

Serge Kakou aborde visuellement le colonialisme, dans une perspective d'information sur cette période plutôt que dans une perspective historique. C'est pourtant à travers la photographie que l'on ressent tout le poids du colonialisme. En effet, les autochtones semblaient être systématiquement définis comme le négatif de l'Occidental. On assemble la composition de l'image pour dénoncer l'indigène comme «feignant, fourbe, pervers, inconséquent et superstitieux » (cité dans Maurel, 1980).

Dans cet ouvrage, l'enjeu et le pouvoir de la photographie auraient pu être discutés plus en profondeur. Il ne fait aucun doute que c'est également à travers l'image que l'on enregistre et interprète l'histoire : un pouvoir qui s'étend non seulement sur la société colonialiste mais également sur ce monde mélanésien où l'appareil photo réussit ce qu'aucun sorcier ne réussit. C'est en prenant une image et en leur donnant une identification que l'on fabrique leur première carte d'identité. Le moment instantané ne se reproduit pas mais le photographe se répète à l'infini même si le sujet change. Tous ces documents qui sont l'œuvre de beaucoup d'auteurs différents semblent sortis d'un seul appareil et d'une seule vision. La photographie nourrit la nostalgie et ravive les souvenirs - privilège de fixer les instants qui se sauvent - , mais traque aussi l'exotisme et favorise l'évasion mentale ; peut-être était-ce le message que l'auteur a voulu nous communiquer?

Emmanuelle CrANE

Doctorante en anthropologie à l'EHEss et en photographie à R.M.I.T. à Melbourne. 